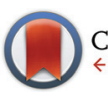

CrossMark

\& click for updates

Cite this: Polym. Chem., 2015, 6 , 3271

Received 30th January 2015,

Accepted 12th March 2015

DOI: 10.1039/c5py00136f

www.rsc.org/polymers

\title{
Selective degradation in aliphatic block copolyesters by controlling the heterogeneity of the amorphous phase $\uparrow$
}

\author{
Veluska Arias, Peter Olsén, Karin Odelius, Anders Höglund and \\ Ann-Christine Albertsson*
}

\begin{abstract}
Controlling the course of the degradation of aliphatic polyesters is a key question when designing new degradable materials. It is shown herein that it is possible to predetermine the degradation path of aliphatic block copolyesters by controlling the heterogeneity of the amorphous phase, which in turn regulates the availability of the hydrolyzable groups in the polyester backbone. To demonstrate these processes, we synthesized a set of degradable materials based on poly(L-lactide) (PLLA), poly( $\varepsilon$-decalactone) $(\mathrm{P} \varepsilon \mathrm{DL})$ and poly $(\varepsilon$-caprolactone) $(\mathrm{PCL})$ with varying compositions. The materials were subjected to hydrolysis for a six months period. The materials composed of PLLA and PEDL exhibited a heterogeneous amorphous phase, whereas the materials composed of PCL and PeDL presented a more homogeneous phase. The kinetics of the degradation indicated that the slowest degradation rate was observed for the more homogeneous compositions. The degradation path of the heterogeneous amorphous phase materials was driven by a random chain scission process, whereas the more homogeneous composition presented a degradation path driven by a more selective chain scission. The confinement of the amorphous phase by the more hydrolytically stable $P \varepsilon D L$ permitted a selective degradation of the available hydrolyzable groups. The random and more selective chain scission processes were further verified by using previously determined molecular modeling based on Monte Carlo procedures. Topographical images and thermal analyses of the materials under different degradation periods correlated with the proposed degradation paths. Detailed insights and the ability to predetermine the degradation pathways of aliphatic polyesters will continue to expand the great potential of renewable materials and their use in specific applications for a future sustainable society.
\end{abstract}

\section{Introduction}

A paradigm shift has occurred in the field of biodegradable polymers, in which the current trend is to design and synthesize polymers with unique chemistry and diverse structures that can tailor their properties towards specific applications while taking into consideration sustainability in their creation. ${ }^{1-3}$ This environmentally responsible approach contemplates the use of materials from renewable feedstock and further engineering of degradation pathways under a reasonable timeframe. However, taking "full advantage" of these materials is hindered by some unsolved problems. Among these challenges is the lack of deep insight and true under-

Department of Fibre and Polymer Technology, KTH Royal Institute of Technology, SE-100 44, Stockholm, Sweden.E-mail: aila@polymer.kth.se

$\dagger$ Electronic supplementary information (ESI) available: ${ }^{1} \mathrm{H}$ NMR spectra of the copolymers after synthesis, logarithmic number averaged molar mass changes, DSC thermograms and compositional changes of the copolymers under hydrolysis in water for six months. See DOI: 10.1039/c5py00136f standing of the degradation paths of the newly designed materials. The most extensively investigated group of degradable polymers is the polyesters, which are polymerized from cyclic lactones and lactides. The abundance of these polymers can be attributed to the immense monomer diversity and synthetic versatility of this group of monomers. ${ }^{4}$

Degradation of these polymers can be both a desirable and undesirable process depending on the time of occurrence. Therefore, by controlling this phenomenon, it is possible to predict the lifetime of materials and tailor them to specific needs. Polyester degradation occurs primarily by chain scission of the main or side chains of the polymer and can be induced by hydrolysis. During hydrolysis, the degradation occurs through cleavage of hydrolytically sensitive bonds in the polymer backbone, finally leading to disintegration of the material. Most polyesters undergo bulk erosion by random scission of the ester group, starting with water uptake, followed by ester bond cleavage, resulting in low molar mass compounds that further autocatalyze the reaction until they are small enough to be released into the medium. ${ }^{5}$ The 
diffusion of the soluble oligomers into the surrounding medium depends on factors such as the polymer's molar mass, the sample thickness and the macromolecular conformation. The size of the sample is very important because, as for polylactide (PLA), the degradation of thick samples in aqueous media ( $>1 \mathrm{~mm}$ ) occurs faster in the inner parts of the samples than near the surface. This leads to a drastic disintegration of the sample due to burst-release of accumulated degradation products. ${ }^{6}$ It has recently also been demonstrated that in thin PLA samples a burst-release of the degradation products can occur if the degradation medium supress the diffusion of the oligomers into the milieu. ${ }^{7}$ The hydrolysis mechanism in polyesters is typically random; however, a more selective hydrolysis is obtained by choosing the distribution of the hydrolyzable groups within the polymer backbone. The first stage of degradation occurs in amorphous regions mainly because water penetration is facilitated through a disordered network of polymer chains, giving rise to more space and mobility for the non-degraded chains, enhancing reorganization, and thus increasing crystallinity. ${ }^{8}$

Because the processes involving polymer erosion are multivariable dependent, the research in this area has used modeling to obtain full comprehension. Several studies have addressed these processes by simulating the degradation course using modeling procedures. ${ }^{9-12}$ Significant insights into the hydrolysis of polyesters have been achieved by simulating the mechanisms behind the degradation of different systems, such as PLA. ${ }^{13,14}$

Today, PLA and poly( $\varepsilon$-caprolactone) (PCL) are among the most studied biodegradable polyesters because of their relatively good mechanical properties, biodegradability and biocompatibility. ${ }^{15-19}$ These polymers present high crystallinity, thus to further extend their range of properties, new polymers such as poly(1,5-dioxepan-2-one) (PDXO) ${ }^{20,21}$ and poly( $\varepsilon$-decalactone) (PعDL $)^{22-24}$ have been suggested and used as comonomers in block sequences to provide new properties. PeDL in combination with PLLA leads to the possibility of producing materials with thermoplastic elastomeric properties because of the limited secondary interactions between PLLA and PeDL resulting in hard and soft domains. ${ }^{24,25}$ Degradable block copolymers have attracted much attention in the scientific community because of the wide range of possibilities to create new materials with tailored mechanical properties and degradability. ${ }^{26-34}$ In terms of degradation, past research has mostly focused on how the degradation path is altered as a function of bulk modifications in the material. ${ }^{28,35-41}$ However, we have previously shown that it is also possible to tailor the degradation path of PLLA-based materials by means of specific interactions between polymer pairs responsible for blend compatibility. ${ }^{41,42}$ In light of this finding, to expand this concept into the design of aliphatic block copolymers, the effect of compatibility between the comonomers in the composition on the degradation path of the material needs to be understood. Our aim is to design a degradation model of aliphatic block copolymers based on the notion that the interaction between the cocomponents would influence the compatibility of the amorphous phase and hence the path of degradation. It is well documented that degradation begins in the amorphous regions; hence, by controlling this first step of degradation it should be possible to predetermine the entire path of the degradation. By choosing comonomers with differences in crystallinity and hydrolytic stability, we hypothesize that this would lead to an amorphous phase with diverse heterogeneity that will influence the availability of the hydrolyzable groups and, consequently, the path of degradation. To achieve this goal, we have carefully chosen and synthesized a series of different block copolymers with various block compositions using PLA, PCL and PeDL as the comonomers.

\section{Results and discussion}

The key question when using degradable polymers is how to predict and control the course of degradation. This will enable the production of renewable and degradable materials with tuned degradability for specific applications. Herein, we have prepared and chosen a group of materials with clear differences in molecular structure (Fig. 1) and physical properties, with a heterogeneous or more homogeneous amorphous phase, to influence the selective hydrolysis of aliphatic polyesters. The copolymer microstructure was determined from the chemical shifts of the carbonyl carbons in ${ }^{13} \mathrm{C} N M R$ spectra (Fig. 2). The sequential monomer addition of LLA and $\mathrm{CL}$ to the macroinitiator of $\varepsilon D L$ (Scheme 1) yielded pure triblock and diblock copolymers, indicating low degree of transesterification. Furthermore, end-groups detected in the ${ }^{1} \mathrm{H}$ NMR spectra of the copolymers indicated that transesterification reactions leading to cyclization were discouraged (Fig. S5 and $\mathrm{S} 6 \dagger)$. The properties of the materials before hydrolysis are presented in Table 1 . The material IDs are represented by the polymers and the compositions of the initial feed of each block in the composition. The films used for hydrolysis could show differences between the bottom and top surfaces due to the fabrication method. It has been reported that the film surface in contact with the glass surface could be smoother

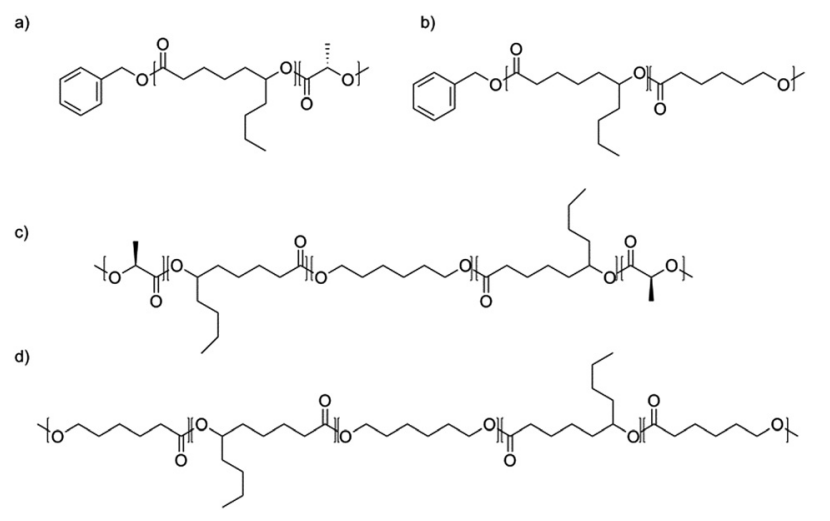

Fig. 1 Molecular structure of (a) $\mathrm{P} \varepsilon D L_{50} \mathrm{PLLA}_{50}$, (b) $\mathrm{PeDL}_{50} \mathrm{PCL}_{50}$, (c) $\mathrm{PLLA}_{33} \mathrm{P}_{\varepsilon} \mathrm{LL}_{33} \mathrm{PLLA}_{33}$ and (d) $\mathrm{PCL}_{33} \mathrm{P}_{\varepsilon} \mathrm{DL}_{33} \mathrm{PCL}_{33}$ after synthesis. 


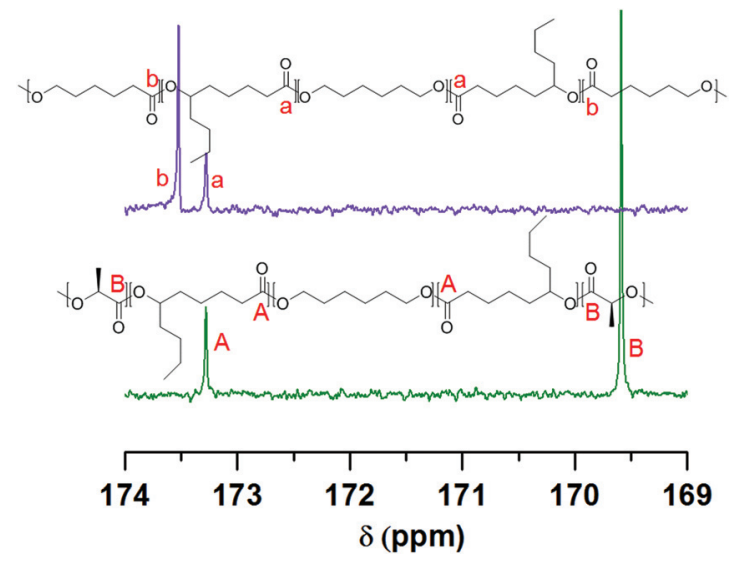

Fig. $2{ }^{13} \mathrm{C}$ NMR spectra of the carbonyl region with peak designation of $\mathrm{PCL}_{33} \mathrm{PeDL}_{33} \mathrm{PCL}_{33}$ (top) and $\mathrm{PLLA}_{33} \mathrm{PeDL}_{33} \mathrm{PLLA}_{33}$ (bottom) after synthesis.

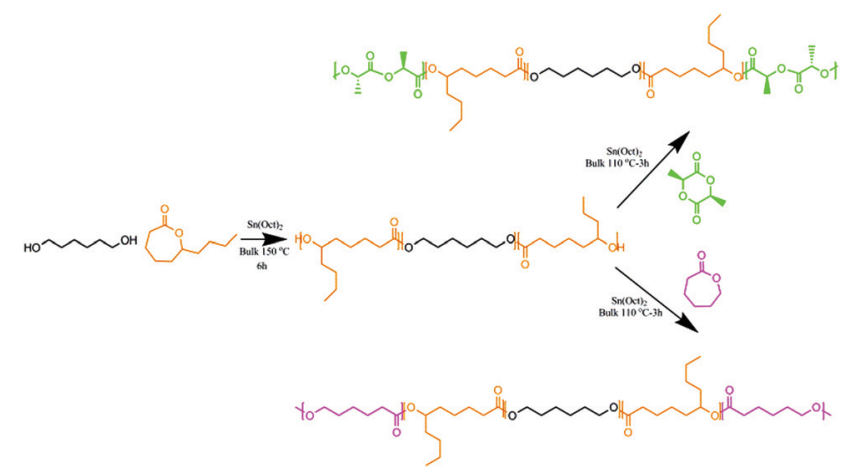

Scheme 1 Depiction of the monomer addition strategy for the copolymerization of $\varepsilon$-Decalactone and L-Lactide, and of $\varepsilon$-Decalactone and $\varepsilon$-Caprolactone using $\mathrm{Sn}(\mathrm{Oct})_{2}$ as catalyst and 1,6-hexanediol as initiator.

than the surface in contact with the air. This affects the wettability and further surface interaction with for example the adherence of microorganisms. ${ }^{43}$ However, this does not affect the bulk properties of the materials which are the changes report in the present article.
The composition of the copolymers was close to the theoretical values prior to synthesis. The materials presented molar masses in the same range with relatively low dispersity $(D)$ values between 1.2 and 1.3. However, dissimilarities were perceived between the two methods used for the molar mass calculation. SEC differentiates the polymers by their hydrodynamic volume and when using polystyrene standards, as in our case, has been reported to overestimate the molar mass of common linear polyesters. ${ }^{44}$ In the case of PeDL, there is an underestimation of the molar mass by SEC using polystyrene standards as a result of suppression of the polymer chain hydrodynamic volume by the solvent polarity. ${ }^{24} \mathrm{PeDL}$ is completely amorphous with a $T_{\mathrm{g}}$ of $-54{ }^{\circ} \mathrm{C}$. The materials with PEDL and PLLA in the composition, i.e., PعDL ${ }_{50} \mathrm{PLLA}_{50}$ and $\mathrm{PLLA}_{33} \mathrm{PeDL}_{33} \mathrm{PLLA}_{33}$, demonstrated two separated amorphous phases for both compositions with the presence of two $T_{\mathrm{g}} \mathrm{S}$ corresponding to the PeDL and PLLA segments at -49 and $48{ }^{\circ} \mathrm{C}$, and -52 and $53{ }^{\circ} \mathrm{C}$, respectively. These compositions showed crystallization induced by the semicrystalline PLLA block. Alternatively, the copolymers composed of PeDL and $\mathrm{PCL}$, i.e., $\mathrm{P}_{\mathrm{DDL}} \mathrm{L}_{50} \mathrm{PCL}_{50}$ and $\mathrm{PCL}_{33} \mathrm{P}_{\varepsilon D L} \mathrm{PL}_{33} \mathrm{PCL}_{33}$, exhibited one amorphous phase for both compositions with the presence of one $T_{\mathrm{g}}$ at -55 and $-57^{\circ} \mathrm{C}$, respectively. This indicates that the two components form a more homogeneous amorphous region opposed to when both PEDL and PLLA are present in the composition. The increased homogeneity in the amorphous regions in the PCL and PeDL triblock polymer was confirmed by DMA analysis with the presence of one $T_{\mathrm{g}}$ at $-43{ }^{\circ} \mathrm{C}$, that is lower than the $T_{\mathrm{g}}$ at $-46^{\circ} \mathrm{C}$ for pure PCL (Fig. S1 $\dagger$ ). The dissimilar $T_{\mathrm{g}}$ values obtained from DSC and DMA analysis, have been reported to differ between $10-30{ }^{\circ} \mathrm{C}$ due to the variations in experimental details such as heating rate for DSC and frequency for DMA. ${ }^{45}$ The latter materials showed crystallization behavior induced by the semicrystalline PCL block. The $T_{\mathrm{g}}$ of these copolymers with miscible comonomers was then theoretically calculated according to the Fox equation (eqn (4)), obtaining a $T_{\mathrm{g}}$ of -57 and $-58{ }^{\circ} \mathrm{C}$ for $\mathrm{PeDL}_{50} \mathrm{PCL}_{50}$ and $\mathrm{PCL}_{33} \mathrm{PeDL}_{33} \mathrm{PCL}_{33}$, respectively. The systems were subsequently hydrolyzed in water for six months, during which time, changes in molar mass, thermal properties, topography, mass loss and $\mathrm{pH}$ were determined at different time periods.

Table 1 Molar mass, dispersity and copolymer composition of the materials before hydrolysis

\begin{tabular}{lllllrr}
\hline Material ID & $\begin{array}{l}\text { Copolymer block } \\
\text { composition }^{a}\end{array}$ & $\begin{array}{l}\text { Copolymer block } \\
\text { composition }^{b}\end{array}$ & $M_{\mathrm{n}}{ }^{b}(\mathrm{Da})$ & $M_{\mathrm{n}}{ }^{c}(\mathrm{Da})$ & $\Xi^{c}$ & $T_{\mathrm{g}}{ }^{d}\left({ }^{\circ} \mathrm{C}\right)$ \\
\hline PeDL $_{100}$ & 1 & - & - & 30300 & 1.2 & $-54 \pm 1$ \\
PLLA $_{100}$ & 1 & - & - & 86000 & 1.3 & $52 \pm 0$ \\
PCL $_{100}$ & 1 & - & - & 63900 & 1.2 & $-58 \pm 0$ \\
PeDL $_{50}$ PLLA $_{50}$ & $1: 1$ & $1: 0.7$ & 57500 & 28600 & 1.1 & $-49 \pm 1 ; 48 \pm 1$ \\
PeDL $_{50}$ PCL $_{50}$ & $1: 1$ & $1: 0.9$ & 74900 & 44200 & 1.3 & $-55 \pm 1$ \\
PLLA $_{33}$ PeDL $_{33}$ PLLA $_{33}$ & $1: 1: 1$ & $1: 1.1: 1$ & 51000 & 45000 & 1.2 & $-52 \pm 1 ; 53 \pm 0$ \\
PCL $_{33}$ PeDL $_{33}$ PCL $_{33}$ & $1: 1: 1$ & $1: 1.1: 1$ & 44100 & 47500 & 1.3 & $-57 \pm 1$ \\
\end{tabular}

${ }^{a}$ Theoretical composition. ${ }^{b}$ Determined from ${ }^{1} \mathrm{H}$ NMR spectra by comparing the integrals of the monomers and initiator. ${ }^{c}$ Determined by SEC using $\mathrm{CHCl}_{3}$ as the eluent and polystyrene standards. ${ }^{d}$ Determined by DSC. Values normalized to the weight fraction. 


\section{Hydrolysis-induced molar mass changes}

The changes in molar mass for the two different systems, i.e., more homogeneous amorphous phase or heterogeneous amorphous phase, presented different behaviors (Fig. 3). Comparing materials with more heterogeneous amorphous phases, i.e., PLLA and PeDL, the diblock PeDL ${ }_{50} \mathrm{PLLA}_{50}$ has a degradation behavior in between that of the triblock $\mathrm{PLLA}_{33^{-}}$ $\mathrm{P}_{2 L_{33}} \mathrm{PLLA}_{33}$ and PLLA 100 . The triblock PLLA ${ }_{33} \mathrm{PeDL}_{33} \mathrm{PLLA}_{33}$, however, exhibits a behavior in between that of the two homopolymers, i.e., $\mathrm{P} \mathrm{DL}_{100}$ and $\mathrm{PLLA}_{100}$. $\mathrm{P} \mathrm{DL}_{100}$ portrayed a slow degradation process where $\sim 90 \%$ of its molar mass remains after six months of degradation. As a consequence, when introducing the amorphous sequence in the middle block of the $\mathrm{PLLA}_{33} \mathrm{PeDL}_{33} \mathrm{PLLA}_{33}$, the molar mass only decreased $\sim 30 \%$ during 133 days of degradation, whereas PLLA $_{100}$ lost $~ 90 \%$ of its molar mass in the same time period. In the case of systems with a more homogenous amorphous phase, i.e., PCL and PeDL-based systems, the largest loss in molar mass was observed for $\mathrm{PCL}_{100}$, which still retained $\sim 70 \%$ of its initial
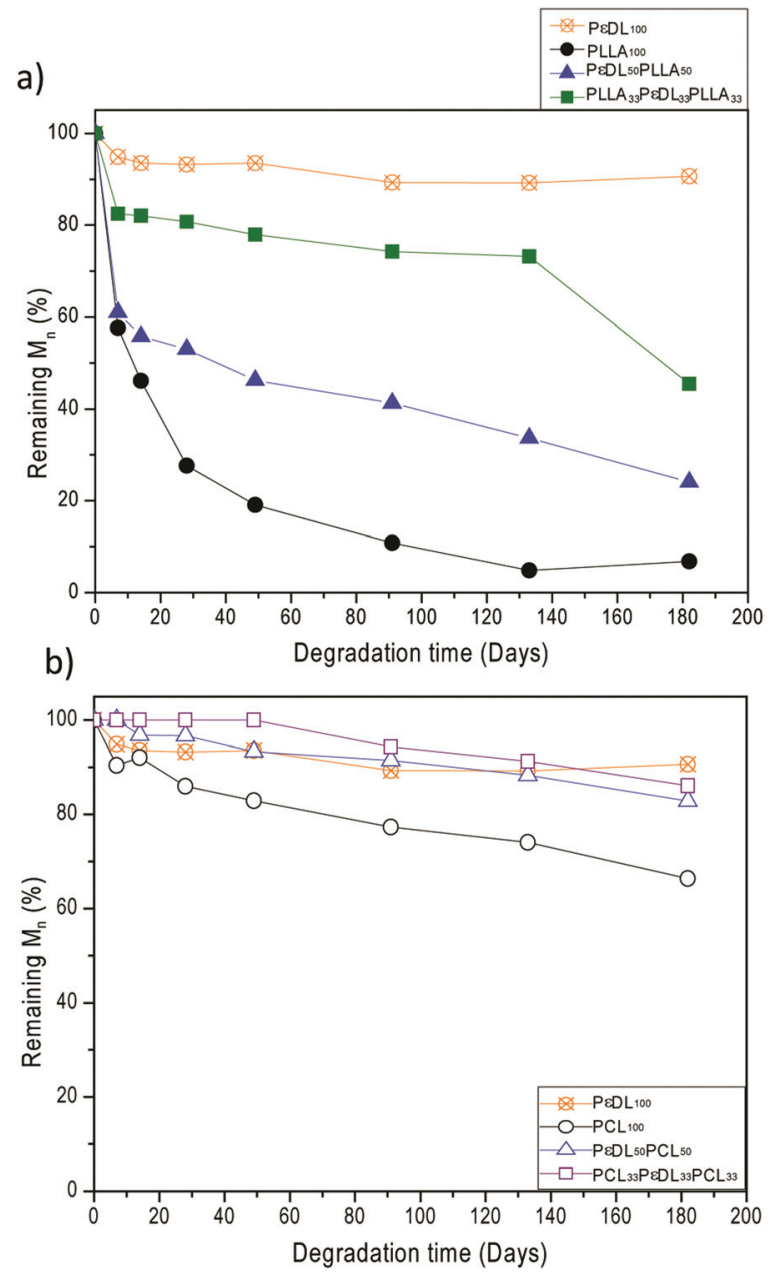

Fig. 3 (a) Molar mass changes of PLLA and PEDL homo-, di-, and triblock copolymers. (b) Molar mass changes of PCL and PعDL homo-, di-, and tri-block copolymers under hydrolysis in water at $37^{\circ} \mathrm{C}$. molar mass after six months of degradation. The addition of PعDL in the composition of PCL-based copolymers, i.e., $\mathrm{PeDL}_{50} \mathrm{PCL}_{50}$ and $\mathrm{PCL}_{33} \mathrm{PeDL}_{33} \mathrm{PCL}_{33}$, led to a slower degradation in comparison to the PCL homopolymer. The molar mass changes were used to follow the kinetics of the degradation by the calculation of the hydrolytic degradation rate constants $(k)$ during the degradation period of six months according to eqn (2).

The $k$ values were estimated from the logarithmic $M_{\mathrm{n}}$ curves relative to the degradation time (Fig. $\mathrm{S} 2 \dagger$ ). ${\mathrm{P} \varepsilon \mathrm{DL}_{100}}_{\text {exhibited }}$ the slowest degradation rate value with one degradation stage and a decrease of $4.7 \times 10^{-6}$ (days $\left.^{-1}\right)$. PCL $_{100}$ and PLLA $_{100}$ were determined to have faster degradation rates of $1.9 \times 10^{-5}$ $\left(\right.$ days $^{-1}$ ) and $2.17 \times 10^{-2}$ (days $^{-1}$ ), respectively. In comparison to $\mathrm{PCL}_{100}$, the degradation of $\mathrm{P} \mathrm{DL}_{100}$ was $\sim 4$ times slower, whereas compared to PLLA 100 , the degradation of $\mathrm{PEDL}_{100}$ was $\sim 4000$ times slower. These results indicate that $\mathrm{PeDL}_{100}$ is more hydrolytically stable than $\mathrm{PCL}_{100}$ and much more hydrolytically stable than PLLA $_{100}$. The fast degradation of PLLA 100 was comparable to our previous results on hydrolysis of PLLA materials in the first stage of degradation. ${ }^{41,46}$ In the case of the diblock materials $\mathrm{PeDL}_{50} \mathrm{PLLA}_{50}$ and $\mathrm{PeDL}_{50} \mathrm{PCL}_{50}$, the degradation rates were $9.1 \times 10^{-3}$ and $8.9 \times 10^{-6}$, respectively. With a diblock composition of PeDL and PLLA, the degradation was $\sim 1000$ times faster than for the diblock composed of PeDL and PCL.

The degradation behavior of the diblock composed of PeDL and PCL had a slower degradation rate than the combination of PeDL and PLLA because their respective homopolymers are hydrolytically more stable during the time of hydrolysis. For the triblock composed of PEDL and PLLA, the degradation was much slower compared to that of the PLLA homopolymer, most likely because of the slower degradation of the central PeDL block. A chain scission in a flanking PLLA block in the triblock has less effect on the molar mass than a random chain scission in the PLLA homopolymer because the flanking PLLA blocks are relatively short. Analogously, a chain scission in the PLLA block has a larger effect on the molar mass in the diblock compared to the triblock copolymer. This is the main reason for the difference in hydrolytic stability between the triblock and diblock of the PeDL and PLLA copolymer. The triblock copolymers $\mathrm{PCL}_{33} \mathrm{PeDL}_{33} \mathrm{PCL}_{33}$ and $\mathrm{PLLA}_{33} \mathrm{P} \mathrm{DL}_{33} \mathrm{PLLA}_{33}$ had a decrease in molar mass at a rate of 0.1 and $0.3 \times 10^{-2}$ $\left(\right.$ days $^{-1}$ ), respectively. The degradation of the PLLA-based triblock was $\sim 3$ times faster than that of the PCL-based triblock. However, the degradation of $\mathrm{PLLA}_{33} \mathrm{P}_{2 \mathrm{DL}}{ }_{33} \mathrm{PLLA}_{33}$ was much slower than PLLA 100 even when PLLA is the major component.

\section{Proposed hydrolysis routes}

The copolymer composition, i.e., the nature of the comonomers, induced changes in the degradation process (Fig. 3). The secondary interactions between the cocomponents amorphous phases as well as the hydrolysis stability of the components drive the path of the hydrolysis as demonstrated by the different hydrolysis patterns. The degradation process then indicates that two different degradation pathways occur during 


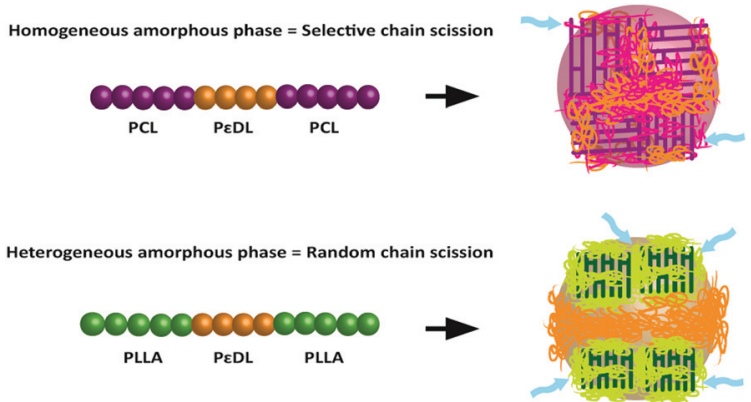

Fig. 4 Proposed degradation routes for copolymers presenting more homogeneous amorphous phase regions, as demonstrated by $\mathrm{PCL}_{33} \mathrm{PEDL}_{33} \mathrm{PCL}_{33}$, and for copolymers with heterogeneous amorphous regions, as demonstrated by $\mathrm{PLLA}_{33} \mathrm{P}_{\varepsilon} \mathrm{DL}_{33} \mathrm{PLLA}_{33}$, under hydrolysis in water at $37^{\circ} \mathrm{C}$.

hydrolysis and depend on the comonomer composition (Fig. 4). The first path occurs when two components interact in a single more homogeneous amorphous phase, which is the case in $\mathrm{PCL}_{33} \mathrm{PeDL}_{33} \mathrm{PCL}_{33}$. The degradation process of this copolymer proceeded such that the more hydrolytically stable PEDL segments protect and render the amorphous regions containing PCL segments less available for degradation. The slow degradation occurs selectively starting from the unprotected amorphous PCL edges until it reaches the center, at which point both amorphous miscible phases will begin to degrade.

The second path occurs when there are two phase-separated components leading to a more heterogeneous amorphous region, which is the case demonstrated by $\mathrm{PLLA}_{33} \mathrm{PEDL}_{33^{-}}$
$\mathrm{PLLA}_{33}$. For this combination, the hydrolysis occurs through a random chain scission of the much faster degrading PLLA amorphous segments. The proposed routes correlate with the degradation profiles obtained by monitoring the remaining molar mass during the hydrolysis (Fig. 3). For $\mathrm{PCL}_{33} \mathrm{PeDL}_{33} \mathrm{PCL}_{33}$, despite the slow degradation process, it follows the line of its respective diblock $\mathrm{P}_{\mathrm{DDL}} \mathrm{L}_{50} \mathrm{PCL}_{50}$. For PLLA $_{33} \mathrm{PeDL}_{33} \mathrm{PLLA}_{33}$, random chain scission is observed as the degradation profile is in between the $\mathrm{PeDL}_{100}$ and the $\mathrm{P}_{\varepsilon L_{50}} \mathrm{PLLA}_{50}$, where the latter resembles the degradation pattern of both PLLA 100 and $\mathrm{PeDL}_{100}$. The degradation line of $\mathrm{PLLA}_{33} \mathrm{PeDL}_{33} \mathrm{PLLA}_{33}$ then follows a degradation path in between that of its predecessors. These degradation patterns are verified by the changes in $T_{\mathrm{g}}$. In Fig. $\mathrm{S} 3, \dagger$ it is shown that the $T_{\mathrm{g}}$ values of $\mathrm{PLLA}_{33} \mathrm{P} \mathrm{DL}_{33} \mathrm{PLLA}_{33}$ decreases for the PLLA segments, whereas it remains stable for the PeDL comonomer, indicating that only the PLLA segments have degraded.

The two systems composed of either a heterogeneous amorphous phase, i.e., PEDL and PLLA, or a more homogeneous amorphous phase, i.e., P\&DL and PCL, are differentiated by the presence of a high or low amount of secondary interactions between the amorphous phases in the copolymer composition. This behavior of the amorphous phases was assessed by the presence of one or two glass transition temperatures in the thermal analysis as well as by atomic force microscopy (AFM). Therefore, to verify the proposed degradation routes, the path of the degradation for the triblocks $\mathrm{PCL}_{33} \mathrm{PeDL}_{33} \mathrm{PCL}_{33}$ and $\mathrm{PLLA}_{33} \mathrm{PeDL}_{33} \mathrm{PLLA}_{33}$ was followed by AFM. AFM phase images were then selected to show the effect of secondary interactions between block cocomponents on the degradation process under different hydrolysis conditions (Fig. 5). For the combination of PLLA and PeDL, PLLA ${ }_{33}$ PEDL $_{33^{-}}$
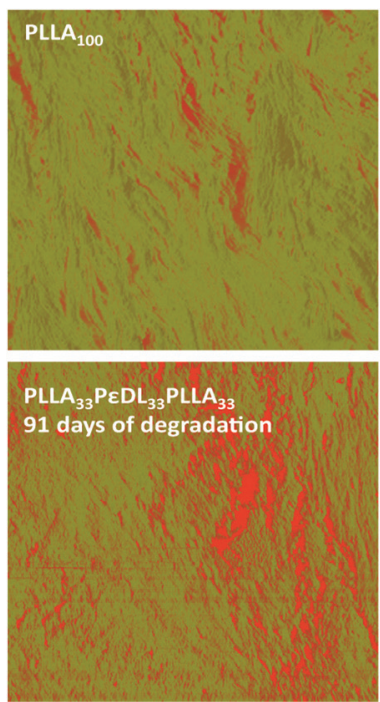
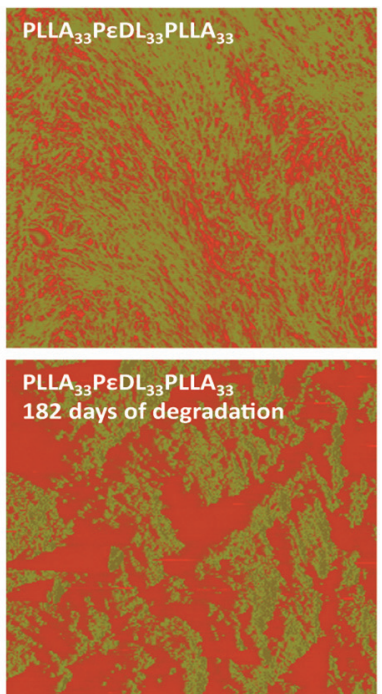
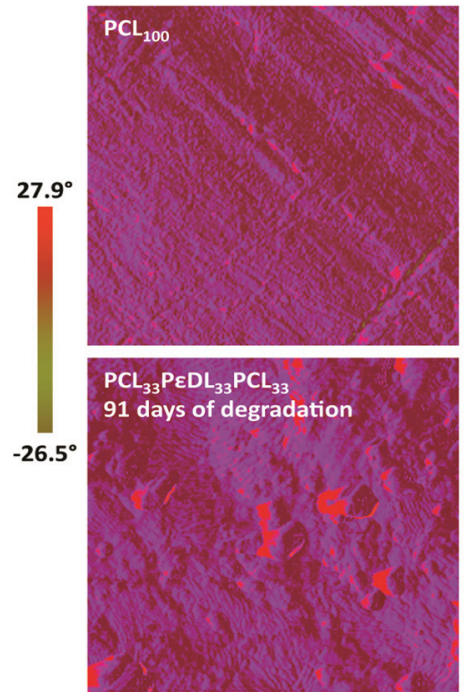

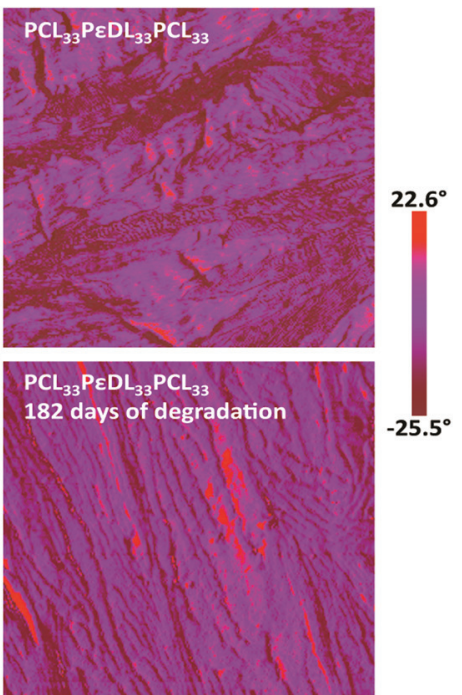

Fig. 5 Representative AFM phase images of the copolymers before and during hydrolysis from the top surface of the films. Left: PLLA $A_{100}$ and $\mathrm{PLLA}_{33} \mathrm{P}_{\varepsilon} \mathrm{DL}_{33} \mathrm{PLLA}_{33}$ before hydrolysis, PLLA ${ }_{33} \mathrm{P}_{\varepsilon} \mathrm{LL}_{33} \mathrm{PLLA}_{33}$ after 91 days of degradation and $\mathrm{PLLA}_{33} \mathrm{P}_{\varepsilon} \mathrm{DL}_{33} \mathrm{PLLA}_{33}$ after 182 days of degradation. Right: $\mathrm{PCL}_{100}$ and $\mathrm{PCL}_{33} \mathrm{P}_{\varepsilon} \mathrm{LL}_{33} \mathrm{PCL}_{33}$ before hydrolysis, $\mathrm{PCL}_{33} \mathrm{P}_{\varepsilon} \mathrm{LL}_{33} \mathrm{PCL}_{33}$ after 91 days of degradation and $\mathrm{PCL}_{33} \mathrm{P \varepsilon DL}_{33} \mathrm{PCL} 33$ after 182 days of degradation. All AFM images were scanned over an area of $5 \mu \mathrm{m} \times 5 \mu \mathrm{m}$. 
PLLA $_{33}$ showed two phases corresponding to crystalline (green phase) and amorphous (orange phase) regions before degradation, where the PeDL domains are spread along the matrix. After 91 days of degradation, the PeDL microdomains appeared to begin merging. Finally, after 182 days of degradation, the PeDL segments are more evident, indicating that only the PLLA segments are degrading and that the phase separation still remains. These results corroborate with the changes in $T_{\mathrm{g}}$ for this formulation (Fig. S3† and 5). In the case of the combination represented by PCL and PeDL, $\mathrm{PCL}_{33} \mathrm{PeDL}_{33} \mathrm{PCL}_{33}$ before degradation demonstrates a single phase where the soft domains (orange phase) seem to coalesce into the PCL matrix (purple phase). After 91 and 182 days of degradation, no significant changes were observed between the phases, confirming the slow degradation process demonstrated by this formulation.

\section{Simple chain scission process using Monte Carlo simulation}

Polymer degradation of aliphatic polyesters primarily occurs by scission of the main or side chains of the polymer. Guaita et $a{ }^{9}{ }^{9}$ investigated Monte Carlo simulations in polymer degradation where volatilization is excluded and it is assumed that the degradation is essentially a chain scission process. In this simulation, it is assumed that macromolecule fragments interact with each other, but the possibility of branched molecule formation is neglected. To verify our proposed degradation routes, where random chain scission occurs when two comonomers are present in a heterogeneous amorphous region, we applied the equations obtained by the above mentioned Monte Carlo simulation to our copolymer systems. This type of process, where the degradation occurs exclusively by chain scissions, is either random or nonrandom. In the simulation, it was found that in the polymer degradation processes, the randomness of the chain scissions can be investigated by following the line of the dispersity $(\nexists)$ as a function of the degradation time. If the scissions are random, the $D$ trend line approaches an equilibrium value of 2 . If the trend does not approach the value of 2 , the scission near the center of the molecule is more likely to occur, i.e., bonds located in specific positions are preferably broken. By applying the following simple scission process relation to our system

$$
S=\frac{\mathrm{DP}_{0}}{\mathrm{DP}_{\mathrm{n}}}-1
$$

where $S$ is the number of scissions per initial molecule, $\mathrm{DP}_{0}$ is the initial number-average degree of polymerization and $\mathrm{DP}_{\mathrm{n}}$ is the number-average degree of polymerization after the scission process, and plotting $D$ as a function of $S$ under the degradation period, similar trends to those achieved by the simulation were obtained. The simulation data for polymers with $D$ values of $\sim 1.1$ gave random scissions when the ratio of $\mathrm{DP}_{0} / \mathrm{DP}_{\mathrm{n}}$ approached a value of 2 . The $D$ lines of the $\mathrm{PLLA}_{33} \mathrm{P}_{2} \mathrm{LL}_{33} \mathrm{PLLA}_{33}$ and $\mathrm{PCL}_{33} \mathrm{PeDL}_{33} \mathrm{PCL}_{33}$ diverge during the degradation process (Fig. 6). In the case of $\mathrm{PLLA}_{33} \mathrm{PeDL}_{33} \mathrm{PLLA}_{33}$, the dispersity values tended to increase, characteristic of a random chain scission process. However,
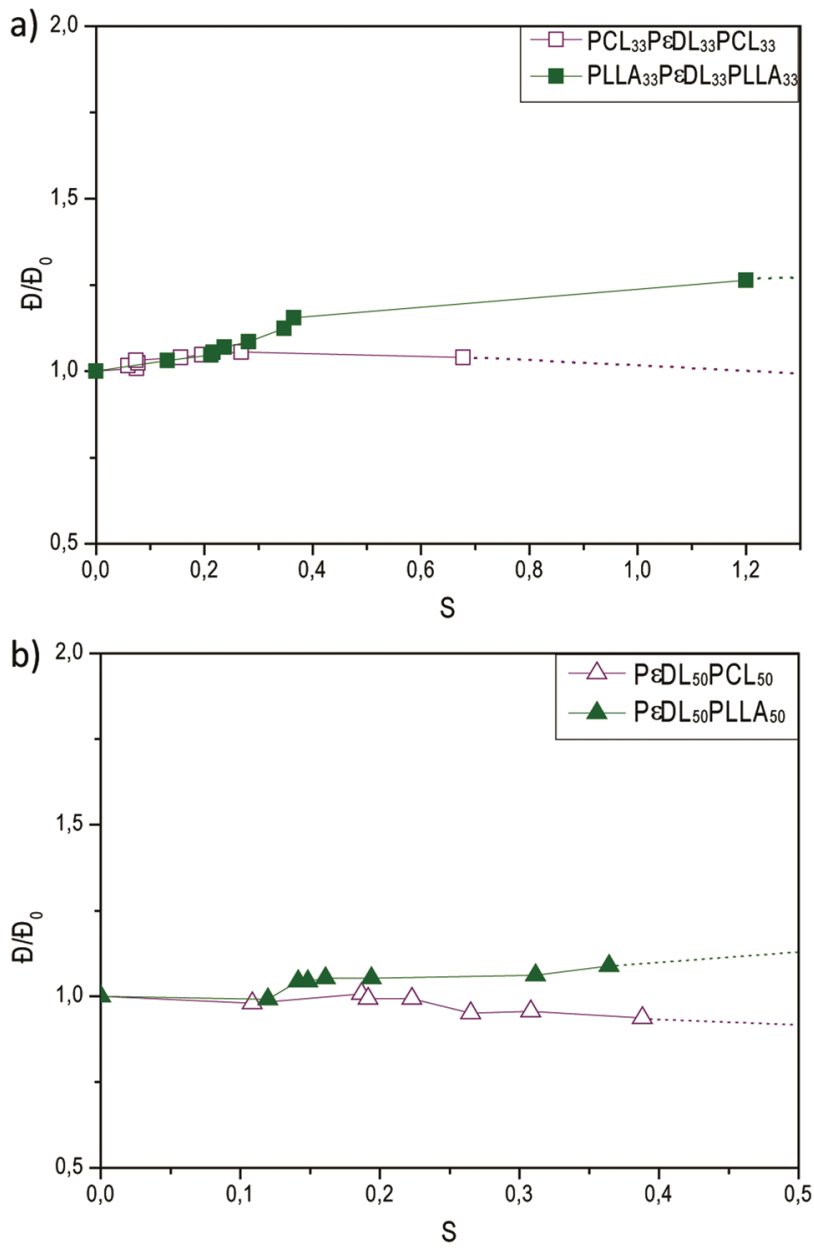

Fig. 6 Relative dispersity values $\left(\Theta / \emptyset_{0}\right)$ normalized to the initial fraction as a function of the number of scissions per original molecule for (a) $\mathrm{PLLA}_{33} \mathrm{PeDL}_{33} \mathrm{PLLA}_{33}$ and $\mathrm{PCL}_{33} \mathrm{PeDL}_{33} \mathrm{PCL}_{33}$; (b) $\mathrm{P}_{\varepsilon} \mathrm{LL}_{50} \mathrm{PLLA}_{50}$ and $\mathrm{P}_{\varepsilon} \mathrm{DL}_{50} \mathrm{PCL}_{50}$ copolymers under hydrolysis in water at $37^{\circ} \mathrm{C}$.

$\mathrm{PCL}_{33} \mathrm{PeDL}_{33} \mathrm{PCL}_{33}$ tends to deviate to lower values, indicating that a more selected scission occurred during the degradation period. This finding correlates to our proposed hydrolysis routes (Fig. 4). The same approach was applied to the diblock copolymers and analogous trends were observed, where the $D$ lines of $\mathrm{PeDL}_{50} \mathrm{PCL}_{50}$ and $\mathrm{PeDL}_{50} \mathrm{PLLA}_{50}$ diverged towards lower and higher values, respectively, demonstrating a controlled and a random chain scission, respectively. In random chain scission, as in PLLA $_{33} \mathrm{PEDL}_{33} \mathrm{PLLA}_{33}$, each ester bond has the same probability of cleavage independent of its length. When bonds located in different positions have unequal probability of being cleaved, nonrandom scission occurs, as shown for $\mathrm{PCL}_{33} \mathrm{P}_{2} \mathrm{DL}_{33} \mathrm{PCL}_{33}$ where the more hydrolytically stable PEDL block protects the PCL segments. It has been demonstrated that for high molar mass samples, one random chain scission has a greater impact on the molar mass than 1000 end or selective scissions, indicating that random chain scissions control molar mass reduction. ${ }^{47}$ This correlates with the faster degradation rate presented by the PEDL- and PLLA-based systems in contrast to the PعDL and PCL-based copolymers. 
Secondary interactions in the amorphous phase influence the hydrolysis process

The interaction between the amorphous phases influences the changes in the glass transition temperature $\left(T_{\mathrm{g}}\right)$ and the crystallinity $\left(w_{\mathrm{c}}\right)$ of the final polymer. The increase in crystallinity may be a result of crystallized oligomers and monomers during the degradation and the faster degradation of the amorphous regions that increase the overall crystallinity. For PeD$\mathrm{L}_{50} \mathrm{PLLA}_{50}$, two clear phases are observed by the appearance of two $T_{\mathrm{g}}$ values corresponding to the PeDL and PLLA components (Table 1). After 91 days of hydrolysis, only the $T_{\mathrm{g}}$ of PeDL was detected and still remained after six months of degradation (Fig. S3†). The melting peak corresponding to the PLLA component broadened and shifted to the right/left during hydrolysis. These results indicate that during degradation, only the PLLA component was affected. In the case of PLLA $_{33} \mathrm{PeDL}_{33} \mathrm{PLLA}_{33}$, two amorphous phases were still observed; however, after 91 days of degradation, the $T_{\mathrm{g}}$ of PeDL became increasingly difficult to detect. This result is likely because of the lower content of PeDL in the copolymer composition of $\mathrm{PLLA}_{33} \mathrm{PeDL}_{33} \mathrm{PLLA}_{33}$ than in $\mathrm{PeDL}_{50} \mathrm{PLLA}_{50}$. The melting peak also decreased and shifted during the degradation process for $\mathrm{PLLA}_{33} \mathrm{PeDL}_{33} \mathrm{PLLA}_{33}$. For the combination of PCL and PeDL, no phase separation was observed. The $T_{\mathrm{g}}$ for $\mathrm{P}_{\varepsilon L_{50}} \mathrm{PCL}_{50}$ decreased during hydrolysis but was still visible after 182 days of degradation. For $\mathrm{PCL}_{33} \mathrm{PeDL}_{33} \mathrm{PCL}_{33}$, only one broad $T_{\mathrm{g}}$ was detected by DSC at $-57^{\circ} \mathrm{C}$ before hydrolysis, which decreased during degradation. Despite this composition presenting a slow degradation, the $T_{\mathrm{g}}$ was difficult to detect at later degradation stages. The detection of $T_{\mathrm{g}}$ in highly crystalline materials by the commonly accepted method of comparing specific crystals heats is difficult because the disorder zones make only a small contribution to the total thermodynamic properties of the material. ${ }^{48}$ The $T_{\mathrm{g}}$ for the PeDL component in $\mathrm{PLLA}_{33} \mathrm{PeDL}_{33} \mathrm{PLLA}_{33}$ does not change during the degradation period, whereas the $T_{\mathrm{g}}$ of the PLLA blocks decreased $\sim 10{ }^{\circ} \mathrm{C}$ during hydrolysis because of the faster degradation rate of the PLLA segments in comparison to the PeDL block. For $\mathrm{PCL}_{33} \mathrm{PeDL}_{33} \mathrm{PCL}_{33}$, only one nearly constant $T_{\mathrm{g}}$ was observed during the degradation period.

The crystallinity of the materials increased during hydrolysis (Fig. 7). This behavior is typical for the heterogeneous degradation process of polyesters, where the amorphous regions are degraded first and the shorter degraded chains can reorganize into a higher crystallinity material. ${ }^{49}$ In the case of $\mathrm{PLLA}_{100}, \mathrm{P}_{\mathrm{DDL}}{ }_{50} \mathrm{PLLA}_{50}$ and $\mathrm{PLLA}_{33} \mathrm{P}_{\mathrm{DDL}} \mathrm{PLLA}_{33}$, the crystallinity increased up to 133 days of degradation and subsequently began to decrease after six months of degradation, indicating that the crystalline regions began to degrade at this point. For the combination of PCL and PEDL, $\mathrm{PCL}_{100}$ underwent an increase followed by a further decrease in its crystallinity, whereas $\mathrm{P} \mathrm{DL}_{50} \mathrm{PCL}_{50}$ and $\mathrm{PCL}_{33} \mathrm{PeDL}_{33} \mathrm{PCL}_{33}$ did not show a decrease in crystallinity in later degradation periods, indicating that the amorphous regions had not fully degraded. PLLA $_{33}$ PeDL $_{33}$ PLLA $_{33}$ has a higher crystallinity than
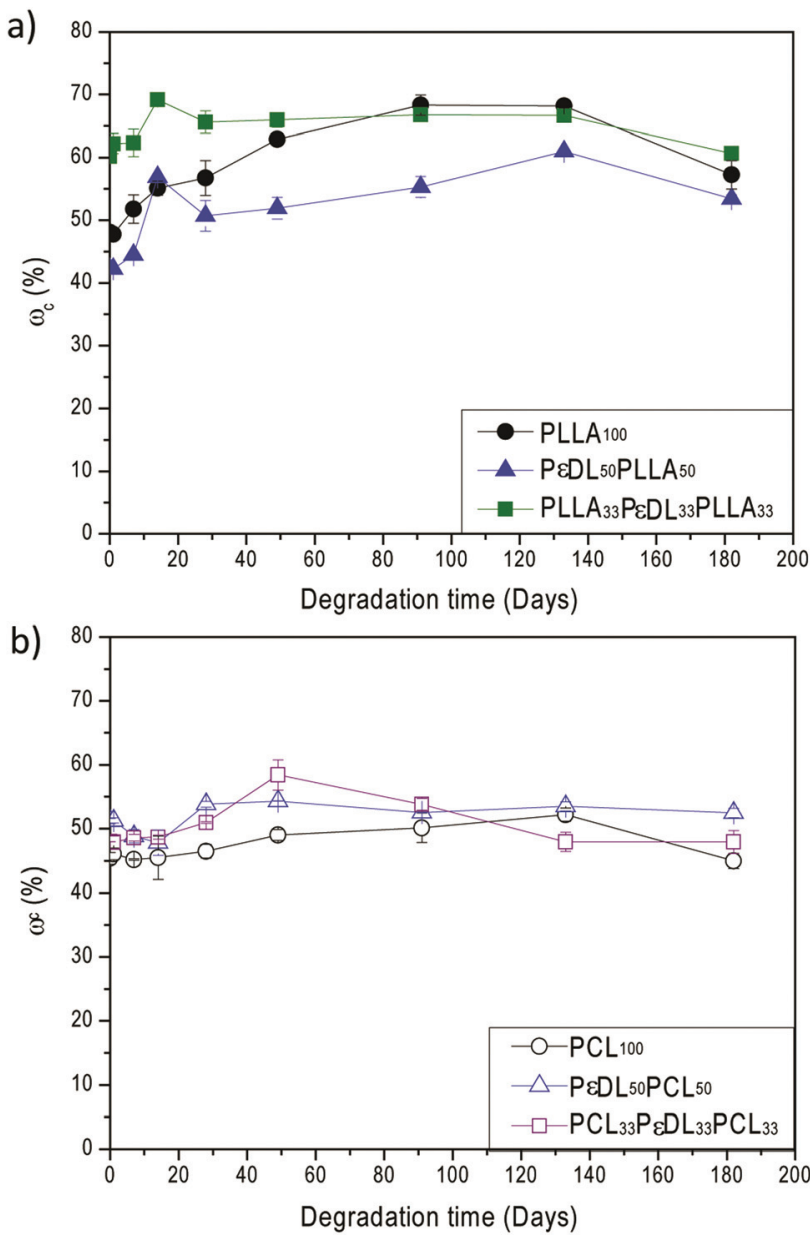

Fig. 7 Crystallinity changes of the materials determined from the $2^{\text {nd }}$ heating scan thermograms after different hydrolysis times in water at $37^{\circ} \mathrm{C}$.

$\mathrm{PCL}_{33} \mathrm{PeDL}_{33} \mathrm{PCL}_{33}$ prior to hydrolysis, nevertheless the degradation rate of the latter was much slower (Fig. 3). This corroborates our hypothesis that factors such as molecular interaction clearly affect the hydrolysis path of the designed polymers.

The morphology of all materials differed due to the dissimilar molecular arrangement in the compositions (Fig. 8). Two distinctive phases were observed in the diblock and triblock copolymers. In the case of $\mathrm{P} \mathrm{DL}_{50} \mathrm{PLLA}_{50}$, the $\mathrm{P} \varepsilon \mathrm{DL}$ phase (dark dots) was dispersed in the PLLA matrix as compared to PLLA $_{100}$. Similarly, small areas of the PeDL phase were seen in $\mathrm{PLLA}_{33} \mathrm{P}_{\mathrm{CDL}} \mathrm{PLLA}_{33}$, most likely because of the smaller amount of PeDL in the total composition compared to PeD$\mathrm{L}_{50}$ PLLA $_{50}$ (Table 1). Analogous TEM patterns have previously been observed for phase-separated copolymers. ${ }^{29,50}$ $\mathrm{P}_{\mathrm{CDL}}{ }_{50} \mathrm{PCL}_{50}$ demonstrated a particular molecular arrangement, with a more homogeneously dispersed PeDL phase in the matrix compared to $\mathrm{PCL}_{100}$. $\mathrm{PCL}_{33} \mathrm{PeDL}_{33} \mathrm{PCL}_{33}$ demonstrated a similar molecular pattern as it analogue $\mathrm{PeDL}_{50} \mathrm{PCL}_{50}$, with smaller domains more homogeneously dis- 


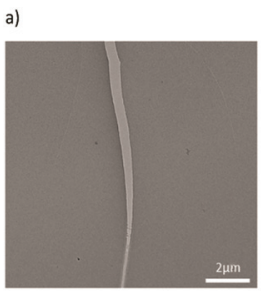

b)

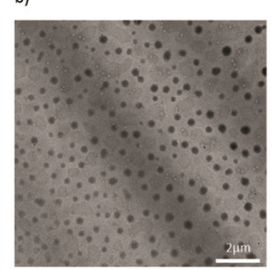

d)

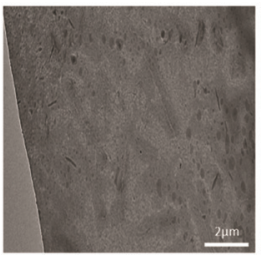

e)



c)

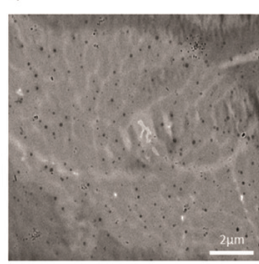

f)



Fig. 8 TEM images of (a) $P_{L L A} 100$, (b) $P_{\varepsilon D L}{ }_{50} P_{L L A}$, (c) PLLA $33^{-}$ $\mathrm{P}_{\varepsilon} L_{33} \mathrm{PLLA}_{33}$ ，(d) $\mathrm{PCL}_{100}$ ，(e) $\mathrm{P}_{\varepsilon} \mathrm{LL}_{50} \mathrm{PCL}_{50}$ and (f) $\mathrm{PCL}_{33} \mathrm{PeDL}_{33} \mathrm{PCL}_{33}$ before hydrolysis.

a)

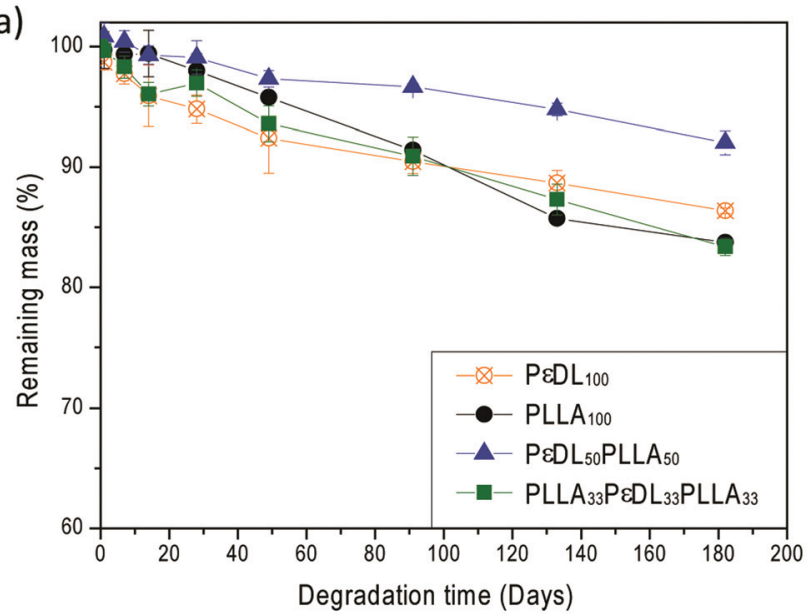

c)

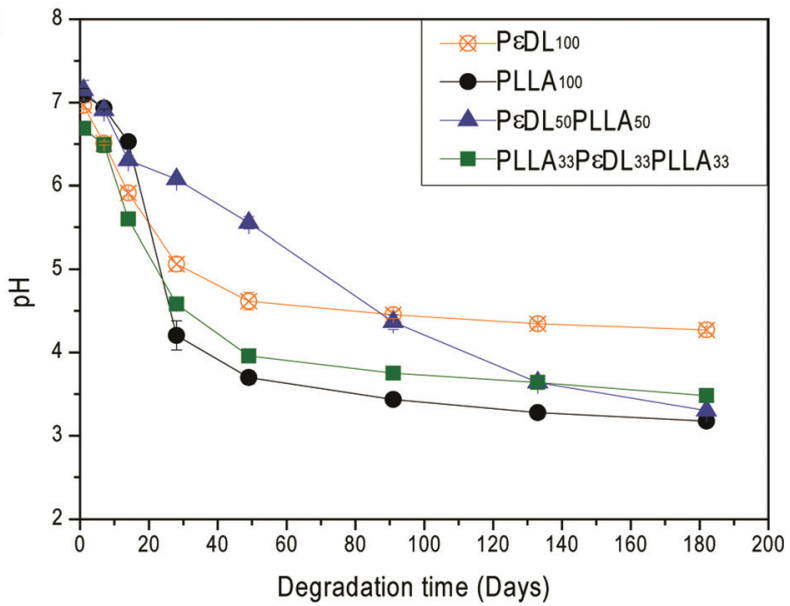

persed in the matrix. The dissimilar morphological structures confirmed the different results obtained by thermal analysis (Table 1, Fig. 7 and S3†) and the topography of the compositions prior to hydrolysis (Fig. 5).

\section{Copolymer compositional changes, mass loss and pH under hydrolysis}

The composition of the copolymers portrayed small variations during the hydrolysis period (Fig. S4 $\dagger$ ). Compositional changes are closely related to mass variations. It is well known that mass changes during degradation are much slower than molar mass changes. This can be explained by the bulk degradation process experienced by most polyesters, where the mass loss begins when the water-soluble oligomers originating from the chain cleavage are small enough to diffuse from the matrix into the degradation medium. ${ }^{6}$

The remaining mass and the $\mathrm{pH}$ were also analyzed for all materials during the degradation process (Fig. 9). For the PeDL- and PCL-based polymers, the mass loss was nearly invariant over the total degradation period, where the highest loss was observed for $\mathrm{P} \mathrm{DL}_{100}$ with $\sim 80 \%$ of its mass remain-
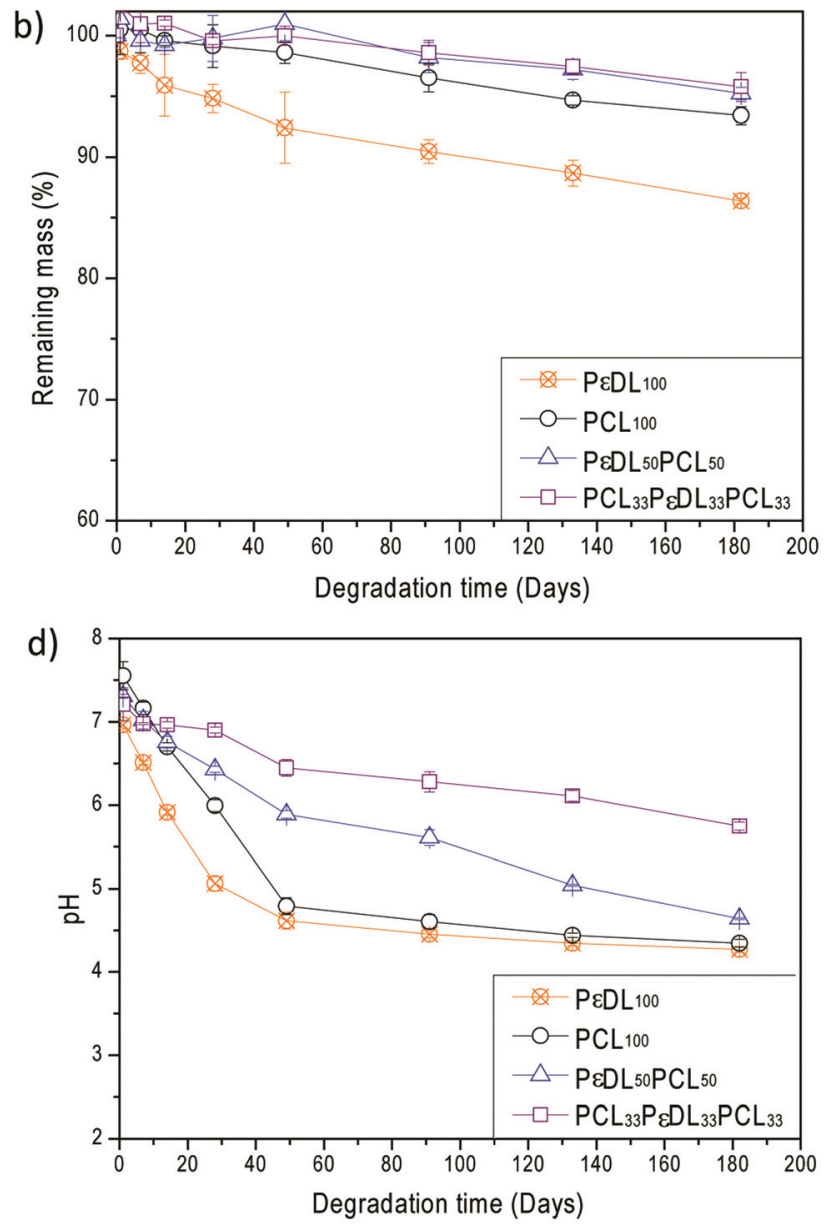

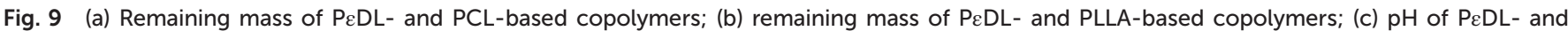
PCL-based copolymers; (d) $\mathrm{pH}$ of PعDL- and PLLA-based copolymers after different hydrolysis times in water at $37^{\circ} \mathrm{C}$. 
ing after 182 days of degradation. This result is most likely because $\mathrm{P} \mathrm{DL}_{100}$ is completely amorphous, thus the chains have more mobility to migrate to the degradation medium. In the case of the PeDL- and PLLA-based systems, the mass loss was also relatively slow in which most of the compositions, $\mathrm{PLLA}_{100}, \mathrm{P} \mathrm{DL}_{100}$ and $\mathrm{PLLA}_{33} \mathrm{PeDL}_{33} \mathrm{PLLA}_{33}$, had a remaining mass of $\sim 80 \%$ after six months of degradation. These results confirm the small compositional variations observed during degradation. The higher mass loss of $\mathrm{P} \mathrm{DL}_{100}$ can be explained by the greater water uptake of the amorphous regions than the crystalline domains. This polymer is completely amorphous (Table 1) and thus has more mobile regions that could drift in the degradation medium without degrading. The $\mathrm{pH}$ of the degradation medium decreased when only a small amount of the monomeric units was released into the water in all cases.

\section{Conclusions}

Predefined hydrolysis paths were achieved by controlling the heterogeneity of the amorphous phase in aliphatic block copolyesters. The availability of the hydrolyzable groups in the polymer backbone was shielded by adding a more hydrolytically stable comonomer to the composition, represented by PeDL. The different copolymers consisted of either semicrystalline PLLA together with the completely amorphous PeDL, or semicrystalline PCL with the completely amorphous PeDL, in different compositions. Copolymers that present weak or strong secondary interactions between its blocks in the amorphous phase were denoted as being heterogeneous or more homogeneous, respectively. Copolymers presenting strong secondary interactions between its blocks were characterized by the presence of a more homogeneous amorphous phase, represented by PCL and PEDL in the composition. Conversely, copolymers presenting poor secondary interactions between its block components had a heterogeneous amorphous phase, represented by PLLA and PeDL in the composition. The slowest degradation rate was observed for the more homogeneous amorphous phase compositions of PCL and PeDL, for the diblock and triblock copolymers. The molar mass changes during degradation indicated that two different degradation paths occurred during hydrolysis depending on the degree of heterogeneity of the amorphous phases. The degradation path for the copolymers with heterogeneous amorphous regions, represented by $\mathrm{PLLA}_{33} \mathrm{PeDL}_{33} \mathrm{PLLA}_{33}$, followed a random chain scission, where the PLLA side blocks were mainly affected during the degradation period. In contrast, the copolymer with a more homogeneous amorphous phase, represented by $\mathrm{PCL}_{33} \mathrm{PEDL}_{33} \mathrm{PCL}_{33}$, degraded via a more selective chain scission starting from the amorphous unprotected PCL segments. Topographical images of the materials under different hydrolysis periods confirmed the random and more selective degradation paths for the heterogeneous, i.e., $\mathrm{PLLA}_{33} \mathrm{P}_{\mathrm{DDL}} \mathrm{PLLA}_{33}$, and the more homogeneous, i.e., $\mathrm{PCL}_{33} \mathrm{P}_{\mathrm{DLL}}{ }_{33} \mathrm{PCL}_{33}$, amorphous phases, respectively. The degradation path was independent from the degree of crystallinity before hydrolysis, indicating that molecular interactions greatly affect the degradation of the materials. The thermal properties, topography and morphology of the materials confirmed the strong and weak secondary interactions between the PCL and PeDL, and the PLLA and PeDL, amorphous phases, respectively. Greater than $80 \%$ mass remained for all materials after six months of degradation. These results reveal how the manipulation of the amorphous phase, in aliphatic polyesters, greatly influences the degradation process. This knowledge will engender a new tool to tailor the degradation process in pursuit of greater control over polymeric structures on the molecular level. And by this, the opportunities to design degradable materials to meet specific applications will increase while at the same satisfying environmental awareness demands.

\section{Experimental}

\section{Materials}

The monomer L-Lactide (LA, Boehringer Ingelheim, France) was purified by recrystallization three times in dry toluene, whereas $\varepsilon$-Decalactone ( $\varepsilon$-DL, Sigma-Aldrich, Sweden) and $\varepsilon$-Caprolactone (CL, Sigma-Aldrich, Sweden) were dried over calcium hydride $\left(\mathrm{CaH}_{2}\right)$ and distilled under reduced pressure before use. Depending on the desired block-structure, 1,6hexanediol (Sigma-Aldrich, Sweden) or benzyl alcohol (SigmaAldrich, Sweden) was used as the initiator. Stannous 2-ethylhexanoate ( $\mathrm{Sn}(\mathrm{Oct})_{2}$, Sigma-Aldrich, Sweden), which was dried over molecular sieves, was used as the catalyst. The solvents methanol (Fisher Scientific, Sweden), ethanol (Fisher Scientific, Sweden), and chloroform (Fisher Scientific, Sweden) were used as received.

\section{Polymer synthesis}

The synthesis of the polymers was performed in bulk where the monomer, initiator and catalyst were added into the reaction vessels under an inert atmosphere. $\mathrm{Sn}(\mathrm{Oct})_{2}$ was used as the catalyst $\left([\mathrm{M}] /\left[\mathrm{Sn}(\mathrm{Oct})_{2}\right] \approx 100\right)$ and benzyl alcohol was used as the initiator in the synthesis of poly(L-lactide) (PLLA), poly( $\varepsilon$-decalactone) (PeDL) and poly( $\varepsilon$-caprolactone) (PCL) homopolymers. The synthesis of PLLA and PCL was conducted in a thermostatically controlled oil bath at $110{ }^{\circ} \mathrm{C}$ and the reaction time was set to $3 \mathrm{~h}$. In the synthesis of PeDL, the reaction conditions were $150{ }^{\circ} \mathrm{C}$ for $6 \mathrm{~h}$ according to a previously reported procedure. $^{24}$

The synthesis of the block copolymers was performed in two-step polymerizations, were the soft segment was polymerized first and, after complete conversion, the second component was added to form the end-blocks. $\mathrm{Sn}(\mathrm{Oct})_{2}$ was used as the catalyst $([\mathrm{M}] /[\mathrm{Sn}(\mathrm{Oct}) 2] \approx 100)$ and 1,6 -hexanediol and benzyl alcohol were used as the initiators for the triblock and diblock copolymers, respectively. When the reaction was complete, the reaction products were cooled to room temperature, further dissolved in chloroform, and finally precipitated in cold methanol three times. The precipitates were dried under reduced pressure for one week. 


\section{Film preparation}

The materials were dissolved in chloroform $(\sim 6 \% \mathrm{w} / \mathrm{w})$ and casted in glass Petri dishes. The solvent was allowed to evaporate and finally the films were dried under reduced pressure for one week before exposure to hydrolysis.

\section{Hydrolysis}

The copolymers and respective homopolymers were subjected to hydrolytic degradation in water at $37^{\circ} \mathrm{C}$ for six months. Each hydrolyzed sample had an approximate weight of $30 \mathrm{mg}$ $\pm 1 \mathrm{mg}$ and a square shape with dimensions of $1 \mathrm{~cm} \times 1 \mathrm{~cm}$ and $0.200-0.300 \mathrm{~mm}$ thickness. The samples were placed in a vial containing $10 \mathrm{~mL}$ of water sealed with a butyl/PTFE septa and aluminum lid before being finally placed in a thermostatically controlled oven. Triplicate samples of each material were withdrawn from the degradation milieu at predetermined time intervals between 1 day and six months, dried under vacuum for one week, and subjected to various analyses.

\section{Mass loss}

The progress of the degradation was monitored by determining the remaining mass of the samples after each hydrolysis period. After withdrawing the materials from the degradation medium, the samples were dried under reduced pressure. The mass loss was determined by comparing the dry mass of the specimen $\left(m_{\mathrm{d}}\right)$ at the specific time point with the initial mass of the specimen $\left(m_{0}\right)$, according to eqn (1).

$$
\Delta m_{\mathrm{d}}=\frac{m_{\mathrm{o}}-m_{\mathrm{d}}}{m_{0}} \times 100
$$

\section{Size exclusion chromatography (SEC)}

The molar mass and dispersity of the starting materials and after each hydrolysis period were evaluated using a Verotech PL-GPC 50 Plus system with a PL-RI Detector and two Mixed-D $(300 \times 7.5 \mathrm{~mm})$ columns from Varian. The samples were injected with a PL-AS RT Autosampler for PLGPC 50 Plus using chloroform as the mobile phase $\left(1 \mathrm{~mL} \mathrm{~min}^{-1}, 30{ }^{\circ} \mathrm{C}\right)$. Polystyrene standards with a narrow molar mass distribution in the range of 580-400 $000 \mathrm{~g} \mathrm{~mol}^{-1}$ were used for calibration. Corrections for flow rate fluctuations were made using toluene as an internal standard. CirrusTM GPC Software was used to process the data. The kinetics of the degradation was followed assuming an exponential decrease of $M_{\mathrm{n}}$ according to eqn (2). ${ }^{51}$

$$
\ln M_{\mathrm{n}}\left(t_{2}\right)=\ln M_{\mathrm{n}}\left(t_{1}\right)-k t
$$

\section{Nuclear magnetic resonance (NMR)}

${ }^{1} \mathrm{H}$ NMR and ${ }^{13} \mathrm{C}$ NMR spectra were obtained using a Bruker Advance DPX-400 Nuclear Magnetic Resonance spectrometer operating at $400 \mathrm{MHz}$ and $100 \mathrm{MHz}$, respectively. Approximately 10 and $100 \mathrm{mg}$ samples were dissolved in $1 \mathrm{~mL}$ of deuterated chloroform $\left(\mathrm{CDCL}_{3}\right)$ in a $5 \mathrm{~mm}$ diameter sample tube for ${ }^{1} \mathrm{H}$ NMR and ${ }^{13} \mathrm{C}$ NMR, respectively. The composition of the materials was determined using ${ }^{1} \mathrm{H}$ NMR by comparison of the peak intensities of the homopolymers at $\delta_{\text {PLLA }} 5.13 \mathrm{ppm}$, $\delta_{\text {PCL }} 4.05$ and $\delta_{\text {PeDL }} 4.83$ ppm. ${ }^{13} \mathrm{C}$ NMR was used to qualitatively determine the block sequences in the carbonyl region. Non-deuterated chloroform was used as an internal standard $(\delta=7.26 \mathrm{ppm}$ and $\delta=77.0 \mathrm{ppm})$.

\section{Differential scanning calorimetry (DSC)}

The thermal properties of the materials were measured using a DSC (Mettler Toledo DSC 820 module). Approximately $5 \mathrm{mg}$ of the polymer was encapsulated in a $40 \mu \mathrm{L}$ aluminum crucible without a pin. The following temperature program was used: (I) heat from $-70{ }^{\circ} \mathrm{C}$ to $200{ }^{\circ} \mathrm{C}$, (II) cool to $-70{ }^{\circ} \mathrm{C}$, and (III) heat for a second time to $200{ }^{\circ} \mathrm{C}$. The heating and cooling rate was $10{ }^{\circ} \mathrm{C} \mathrm{min}^{-1}$ under a nitrogen atmosphere (nitrogen flow rate of $50 \mathrm{~mL} \mathrm{~min}^{-1}$ ). From the second heating scan, the melting temperature, $T_{\mathrm{m}}$, was noted as the maximum value of the melting peak, and the glass transition temperature, $T_{\mathrm{g}}$, was determined from the midpoint temperature of the glass transition. When determining the crystallinity of the copolymers, it was assumed that the only block contributing to the heat of fusion was the PLLA or the PCL component. The approximate crystallinity of the materials was calculated according to eqn (3).

$$
w_{\mathrm{c}}=\frac{\Delta H_{\mathrm{f}}}{\Delta H_{\mathrm{f}}^{0} w_{\mathrm{f}}} \times 100
$$

where $w_{\mathrm{c}}$ is the degree of crystallinity, $\Delta H_{\mathrm{f}}$ is the heat of fusion of the sample, $\Delta H_{\mathrm{f}}{ }^{0}$ is the heat of fusion of $100 \%$ crystalline polymer and $w_{\mathrm{f}}$ is the weight fraction of the crystalline polymer in the sample. For PLLA and PCL, $\Delta H_{\mathrm{f}}{ }^{o}$ is $93 \mathrm{~J} \mathrm{~g}^{-1}$ (ref. 52) and $139.5 \mathrm{~J} \mathrm{~g}^{-1},{ }^{53}$ respectively. Additionally, in block copolymers where the comonomers are miscible, $T_{\mathrm{g}}$ of the block copolymer can be calculated according to the Fox equation: ${ }^{54}$

$$
\frac{1}{T_{\mathrm{g}}}=\frac{w_{1}}{T_{\mathrm{g} 1}}+\frac{w_{2}}{T_{\mathrm{g} 2}}
$$

where $T_{\mathrm{g}}$ is the glass transition temperature of the block copolymer, $T_{\mathrm{g} 1}$ and $T_{\mathrm{g} 2}$ and $w_{1}$ and $w_{2}$ are the $T_{\mathrm{g}} \mathrm{s}$ and weight fractions of comonomers 1 and 2 , respectively.

\section{pH}

pH measurements of the degradation medium were performed after each hydrolysis period using a calibrated pH-meter containing an $\mathrm{Ag} / \mathrm{AgCl}$ electrode.

\section{Atomic force microscopy (AFM)}

The materials were topographically characterized using a nanoscope IIIa multimode AFM (Digital Instruments, United States) via a $7850 \mathrm{EV}$ scanner. A silicon-etched probe tip (TAP150, Bruker, United States) with a normal spring constant $(k)$ of $5 \mathrm{~N} \mathrm{~m}^{-1}$ and a resonant frequency $\left(f_{\mathrm{o}}\right)$ of $150-200 \mathrm{kHz}$ was used to scan the image in the tapping mode. The surface of the materials was scanned in the range of $1-2 \mathrm{~Hz}$ with a selected maximum sample size $(512 \times 512$ pixels $)$. 


\section{Transmission electron microscopy (TEM)}

The morphology of the copolymers was analyzed using a TEM Hitachi HT 7700 operated at an acceleration voltage of $100 \mathrm{kV}$. Sample solutions at a concentration of $4 \mathrm{mg} \mathrm{mL}^{-1}$ in $\mathrm{CHCl}_{3}$ were casted over 400 mesh copper grids (Ted Pella, Inc. USA) and allowed to dry overnight before analysis.

\section{Dynamic mechanical analysis (DMA)}

Dynamic mechanical analysis of the copolymers prior to degradation was performed with a TA instruments Q800 dynamic mechanical analyser, operated in tensile mode. The specimens were $8 \times 5 \mathrm{~mm}^{2}$ and $\sim 0.2 \mathrm{~mm}$ thick. The temperature program proceeded as follows: equilibrate at $-100{ }^{\circ} \mathrm{C}$ for $5 \mathrm{~min}$ before heating to $50{ }^{\circ} \mathrm{C}$ at a rate of $5{ }^{\circ} \mathrm{C} \mathrm{min}^{-1}$. The oscillation frequency was maintained at $1 \mathrm{~Hz}$ with constant amplitude of $10.0 \mu \mathrm{m}$.

\section{Acknowledgements}

The authors acknowledge the Swedish Research Council, VR (Grant ID: A0347801) and the ERC Advance Grant, PARADIGM (Grant agreement no.: 246776) for their financial support of this work.

\section{Notes and references}

1 P. Olsen, J. Undin, K. Odelius and A.-C. Albertsson, Polym. Chem., 2014, 5, 3847-3854.

2 W. Zhao, L. Glavas, K. Odelius, U. Edlund and A.-C. Albertsson, Chem. Mater., 2014, 26, 4265-4273.

3 P. Olsén, K. Odelius and A.-C. Albertsson, Macromolecules, 2014, 47, 6189-6195.

4 L. Fambri, C. Migliaresi, K. Kesenci and E. Piskin, in Integrated Biomaterials Science, ed. R. Barbucci, Springer, US, 2002, ch. 4, pp. 119-187.

5 C. G. Pitt, F. I. Chasalow, Y. M. Hibionada, D. M. Klimas and A. Schindler, J. Appl. Polym. Sci., 1981, 26, 3779-3787.

6 M. Vert, S. Li, H. Garreau, J. Mauduit, M. Boustta, G. Schwach, R. Engel and J. Coudane, Angew. Makromol. Chem., 1997, 247, 239-253.

7 J. Rydz, G. Adamus, K. Wolna-Stypka, A. Marcinkowski, M. Misiurska-Marczak and M. M. Kowalczuk, Polym. Degrad. Stab., 2013, 98, 316-324.

8 A.-C. Albertsson, J. Macromol. Sci., Part A: Pure Appl. Chem., 1993, A30, 757-765.

9 M. Guaita, O. Chiantore and M. P. Luda, Macromolecules, 1990, 23, 2087-2092.

10 A. Tayal and S. A. Khan, Macromolecules, 2000, 33, 94889493.

11 A. M. Emsley and R. J. Heywood, Polym. Degrad. Stab., 1995, 49, 145-149.

12 S. Akbulatov, Y. Tian, E. Kapustin and R. Boulatov, Angew. Chem., Int. Ed., 2013, 52, 6992-6995.
13 Y. Mohammadi and E. Jabbari, Macromol. Theory Simul., 2006, 15, 643-653.

14 A. Gleadall, J. Pan, M.-A. Kruft and M. Kellomäki, Acta Biomater., 2014, 10, 2223-2232.

15 L. S. Nair and C. T. Laurencin, Prog. Polym. Sci., 2007, 32, 762-798.

16 X. Guo, C. Shi, G. Yang, J. Wang, Z. Cai and S. Zhou, Chem. Mater., 2014, 26, 4405-4418.

17 O. Stoilova, C. Jérôme, C. Detrembleur, A. MouithysMickalad, N. Manolova, I. Rashkov and R. Jérôme, Chem. Mater., 2006, 18, 4917-4923.

18 Q. Peng, K. Mahmood, Y. Wu, L. Wang, Y. Liang, J. Shen and Z. Liu, Green Chem., 2014, 16, 2234-2241.

19 Y. Ikada and H. Tsuji, Macromol. Rapid Commun., 2000, 21, 117-132.

20 T. Mathisen and A. C. Albertsson, Macromolecules, 1989, 22, 3838-3842.

21 M. Ryner and A.-C. Albertsson, Biomacromolecules, 2002, 3, 601-608.

22 J.-O. Lin, W. Chen, Z. Shen and J. Ling, Macromolecules, 2013, 46, 7769-7776.

23 L. Glavas, P. Olsén, K. Odelius and A.-C. Albertsson, Biomacromolecules, 2013, 14, 4150-4156.

24 P. Olsén, T. Borke, K. Odelius and A.-C. Albertsson, Biomacromolecules, 2013, 14, 2883-2890.

25 M. T. Martello, D. K. Schneiderman and M. A. Hillmyer, ACS Sustainable Chem. Eng., 2014, 2, 2519-2526.

26 M. Hakkarainen, A. Höglund, K. Odelius and A.-C. Albertsson, J. Am. Chem. Soc., 2007, 129, 6308-6312.

27 S. Målberg, A. Höglund and A.-C. Albertsson, Biomacromolecules, 2011, 12, 2382-2388.

28 A. Höglund, S. Målberg and A.-C. Albertsson, Macromol. Biosci., 2012, 12, 260-268.

29 D. A. Olson, L. Chen and M. A. Hillmyer, Chem. Mater., 2007, 20, 869-890.

30 T. Lebarbe, M. Neqal, E. Grau, C. Alfos and H. Cramail, Green Chem., 2014, 16, 1755-1758.

31 C. Vaida, H. Keul and M. Moeller, Green Chem., 2011, 13, 889-899.

32 B. Vaisman, D. E. Ickowicz, E. Abtew, M. Haim-Zada, A. Shikanov and A. J. Domb, Biomacromolecules, 2013, 14, 1465-1473.

33 J. Hua, K. Gebarowska, P. Dobrzynski, J. Kasperczyk, J. Wei and S. Li, J. Polym. Sci., Part A: Polym. Chem., 2009, 47, 3869-3879.

34 R. T. Martin, L. P. Camargo and S. A. Miller, Green Chem., 2014, 16, 1768-1773.

35 X. Li, S. Kondo, U.-I. Chung and T. Sakai, Chem. Mater., 2014, 26, 5352-5357.

36 J. Li, R. M. Stayshich and T. Y. Meyer, J. Am. Chem. Soc., 2011, 133, 6910-6913.

37 D. A. Olson, S. E. A. Gratton, J. M. DeSimone and V. V. Sheares, J. Am. Chem. Soc., 2006, 128, 13625-13633.

38 U. Witt, M. Yamamoto, U. Seeliger, R.-J. Müller and V. Warzelhan, Angew. Chem., Int. Ed., 1999, 38, 14381442. 
39 S. Yang, S. A. Madbouly, J. A. Schrader, G. Srinivasan, D. Grewell, K. G. McCabe, M. R. Kessler and W. R. Graves, Green Chem., 2015, 17, 380-393.

40 Y. Han, Z. Fan, Z. Lu, Y. Zhang and S. Li, Macromol. Mater. Eng., 2012, 297, 128-135.

41 V. Arias, A. Höglund, K. Odelius and A.-C. Albertsson, Biomacromolecules, 2014, 15, 391-402.

42 S. R. Andersson, M. Hakkarainen and A.-C. Albertsson, Biomacromolecules, 2010, 11, 3617-3623.

43 Z. G. Tang, R. A. Black, J. M. Curran, J. A. Hunt, N. P. Rhodes and D. F. Williams, Biomaterials, 2004, 25, 4741-4748.

44 H. R. Kricheldorf and S. Eggerstedt, Macromol. Chem. Phys., 1998, 199, 283-290.

45 P. J. Achorn and R. G. Ferrillo, J. Appl. Polym. Sci., 1994, 54, 2033-2043.
46 A. Höglund, K. Odelius and A.-C. Albertsson, ACS Appl. Mater. Interfaces, 2012, 4, 2788-2793.

47 C. Shih, J. Controlled Release, 1995, 34, 9-15.

48 F. C. Stehling and L. Mandelkern, Macromolecules, 1970, 3, 242-252.

49 M. Vert, J. Mauduit and S. Li, Biomaterials, 1994, 15, 12091213.

50 E. M. Frick, A. S. Zalusky and M. A. Hillmyer, Biomacromolecules, 2003, 4, 216-223.

51 C. G. Pitt and G. Zhong-wei, J. Controlled Release, 1987, 4, 283-292.

52 E. W. Fischer, H. J. Sterzel and G. Wegner, Kolloid Z. Z. Polym., 1973, 251, 980-990.

53 V. Crescenzi, G. Manzini, G. Calzolari and C. Borri, Eur. Polym. J., 1972, 8, 449-463.

54 T. G. Fox, Bull. Am. Phys. Soc., 1956, 1, 123. 